\title{
RNAi-mediated silencing of the HD-Zip gene HD20 in Nicotiana attenuata affects benzyl acetone emission from corollas via ABA levels and the expression of metabolic genes
}

\author{
Delfina A Ré ${ }^{1,2}$, Brenda Raud', Raquel L Chan², lan T Baldwin and Gustavo Bonaventure ${ }^{1 *}$
}

\begin{abstract}
Background: The N. attenuata HD20 gene belongs to the homeodomain-leucine zipper (HD-Zip) type I family of transcription factors and it has been previously associated with the regulation of ABA accumulation in leaves and the emission of benzyl acetone (BA; 4-phenyl-2-butanone) from night flowers. In this study, N. attenuata plants stably reduced in the expression of HD20 (ir-hd20) were generated to investigate the mechanisms controlling the emission of BA from night flowers.
\end{abstract}

Results: The expression of HD20 in corollas of ir-hd20 plants was reduced by 85 to $90 \%$ compared to wild-type plants (WT) without affecting flower morphology and development. Total BA emitted from flowers of ir-hd20 plants was reduced on average by $60 \%$. This reduction occurred mainly at the late phase of BA emission and it was correlated with 2-fold higher levels of ABA in the corollas of ir-hd20 plants. When a 2-fold decline in ABA corolla levels of these plants was induced by salt stress, BA emissions recovered to WT levels. Supplying ABA to WT flowers either through the cuticle or by pedicle feeding reduced the total BA emissions by 25 to $50 \%$; this reduction occurred primarily at the late phase of emission (similar to the reduction observed in corollas of ir-hd20 plants). Gene expression profiling of corollas collected at $12 \mathrm{pm}$ (six hours before the start of BA emission) revealed that 274 genes changed expression levels significantly in ir-hd20 plants compared to WT. Among these genes, more than $35 \%$ were associated with metabolism and the most prominent group was associated with the metabolism of aromatic compounds and phenylpropanoid derivatives.

Conclusions: The results indicated that regulation of ABA levels in corollas is associated with the late phase of BA emission in N. attenuata plants and that HD20 affects this latter process by mediating changes in both ABA levels and metabolic gene expression.

\section{Background}

Many plant species emit floral scents as long-distance attraction cues for pollinators, in particular moths, that search and visit flowers at night. The attraction of moths and other animal pollinators have important implications for plant reproductive success; floral scents are important in determining seed or fruit set in non-selfing plants and the frequency of outcross in selfing plants [1-4]. Thus, the biosynthesis and release of floral scents have important

\footnotetext{
* Correspondence: gbonaventure@ice.mpg.de

'Department of Molecular Ecology, Max Planck Institute for Chemical Ecology, 07745, Jena, Germany

Full list of author information is available at the end of the article
}

ecological and agricultural implications and the molecular mechanisms underlying the regulation of floral scent production are now beginning to be unraveled $[5,6]$.

Floral scents are usually complex mixtures of small volatile molecules and the most prevalent compounds in these mixtures are monoterpenoids, sesquiterpenoids, phenylpropanoids, benzenoid compounds and fatty acid derivatives [1]. Volatile compounds can be either emitted from several parts of the flower or from a specific floral part [7]. The expression of genes encoding floral scent biosynthetic enzymes is temporally and spatially regulated during flower development [1,8]. In most cases studied so far, the expression of these genes correlates 
with the emission of the corresponding volatile compound indicating that these volatiles are de novo synthesized. Thus, scent production and emission are commonly regulated at the transcriptional level. Similar observations have been made in regard to the induction of genes involved in floral pigment production [9].

Nicotiana attenuata, a night-flowering tobacco that germinates after fires in the Southwestern United States, normally produces flowers that open at night and release benzyl acetone (BA; 4-phenyl-2-butanone) to attract night-active hawk moth pollinators (Manduca quinquemaculata and M. sexta)[10]. N. attenuata is a fully self-compatible species however it produces more than $30 \%$ of its seed from opportunistic out-crossing performed by pollinators $[2,11]$. BA is the main constituent of the $N$. attenuata floral bouquet [10] and it begins to be emitted from the corolla limb in the evening [10,12]. The emission of BA is synchronized with the development of the flower and it starts as the corolla limb opens [12]. At present, the mechanisms that control the biosynthesis and release of BA from corollas are largely unknown. The BA biosynthesis pathway remains elusive, however, the chemical backbone may derived from the shikimate pathway [1] as $N$. attenuata plants with reduced expression of CHALCONE SYNTHASE 1 (CHAL1) are deficient in BA emission [2].

The homeodomain-leucine zipper (HD-Zip) family of transcription factors (TFs) is a plant-specific family of TFs in which the HD and Zip domains are combined in a single polypeptide [13]. The HD is responsible for the specific binding to DNA and the Zip domain acts as a dimerization motif; dimerization is a prerequisite for binding to the phylogenetically conserved target sequence CAAT (A/T) ATTG [14,15]. The HD-Zip family can be divided into four subfamilies (I to IV) according to the sequence similarity of the HD and Zip domains and to additional structural features outside these domains. The function of HDZips type-I has been associated to the regulation of development in response to changes in the environment [13,16-26].

The $N$. attenuata HD20 gene was originally identified as an HD-Zip type-I whose expression is induced by multiple stress-associated stimuli including drought and wounding [27]. In a previous study, we reduced the expression of HD2O by virus induced gene silencing (VIGS) and we demonstrated that $H D 20$ plays not only a positive role in ABA accumulation in leaves during water stress but also in the emission of benzyl acetone (BA) from night flowers [27]. However, the HD20-dependent mechanisms underlying the reduction in $\mathrm{BA}$ emission were not studied. Here we investigated these mechanisms by generating transgenic $N$. attenuata plants stably reduced in the expression of $H D 20$. These plants were characterized by using a combination of volatile, phytohormone and gene expression profiling approaches to investigate in more detail the process of BA emission.

\section{Results}

\section{HD20 mRNA expression in corolla during development}

To investigate in detail the regulation of HD20 mRNA expression during flower and corolla development in WT plants, the levels of this transcript were first quantified at progressive stages of flower development. HD20 mRNA was detected in all flower stages and the levels increased as the flower entered the opened corolla stage (opened corolla flower (OCF); Figure 1a). The expression of HD20 was then quantified during the opening of the corolla, which is a continuous process that starts in the afternoon (between $12 \mathrm{pm}$ and $4 \mathrm{pm}$ ) and completes at 8 to $9 \mathrm{pm}$ (see below). HD2O mRNA levels were quantified at $12 \mathrm{pm}, 4 \mathrm{pm}, 8 \mathrm{pm}$ and $12 \mathrm{am}$. The transcript levels increased steadily during the afternoon to peak at $8 \mathrm{pm}$ and to decline at $12 \mathrm{am}$ (Figure 1b).

\section{Transformation of N. attenuata plants to reduce HD20 expression}

To investigate the mechanisms mediated by HD2O and controlling the emission of BA from flowers, stably transformed $N$. attenuata plants with reduced levels of HD2O expression were generated by inverted-repeat (IR) gene silencing. These lines were named ir- $h d 20$ and two homozygous independently transformed lines (ir-hd20-1 and irhd20-2) carrying a single T-DNA insertion (Additional file 1) were selected and used for all the experiments in this study (Figure 2a; see Materials and Methods for a detailed description about the generation of these plants). The efficiency of gene silencing in the flowers and corolla of these lines was evaluated by the quantification of HD2O transcript levels at different developmental stages. The levels of this transcript were reduced on average between 85 and 95\% (depending on the flower stage and compared to WT plants) with the exception of ir-hd20-1 that showed WT levels of $H D 20$ expression in the closed-bud stage (CB; Figure 2b; univariate ANOVA, $F_{14,44}=26.64 P<0.001$ followed by a LSD post-hoc test $P<0.01$ ). In corollas, the reduction in HD20 mRNA levels was $87 \%$ in ir- $h d 20-1$ and 97\% in ir-hd20-2 lines (Figure 2b). The morphology and growth of ir- $h d 20$ plants were indistinguishable from those of WT (Figure 2a and Additional file 2). Flower morphology, flowering time and time of corolla opening were also similar between ir- $h d 20$ and WT plants (Figures $2 \mathrm{c}, \mathrm{d}$ and Additional file 2). Consistent with $N$. attenuata plants silenced in HD20 expression by VIGS [27], bolting time was delayed in ir-hd20 plants compared to WT (Additional file 2). 


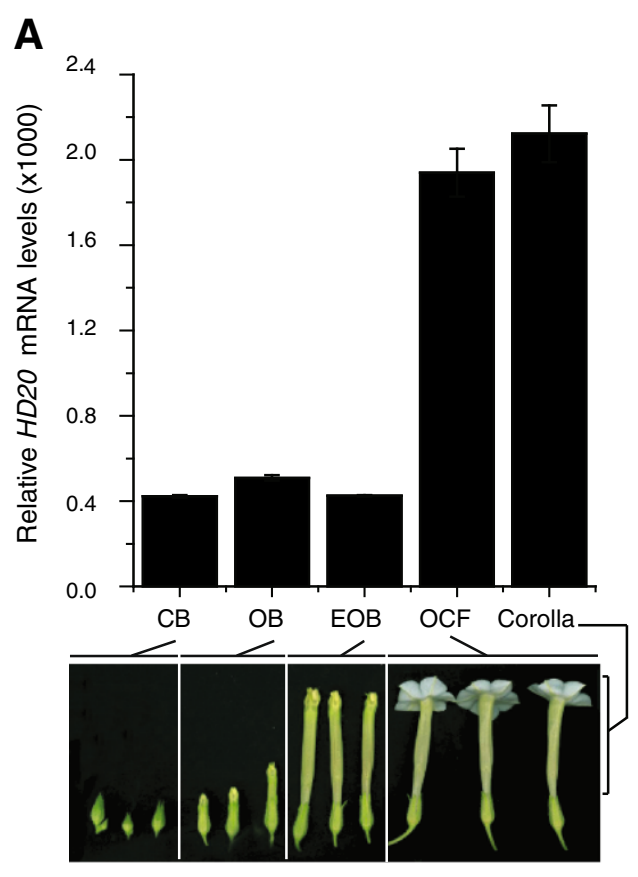

flower developmental stages

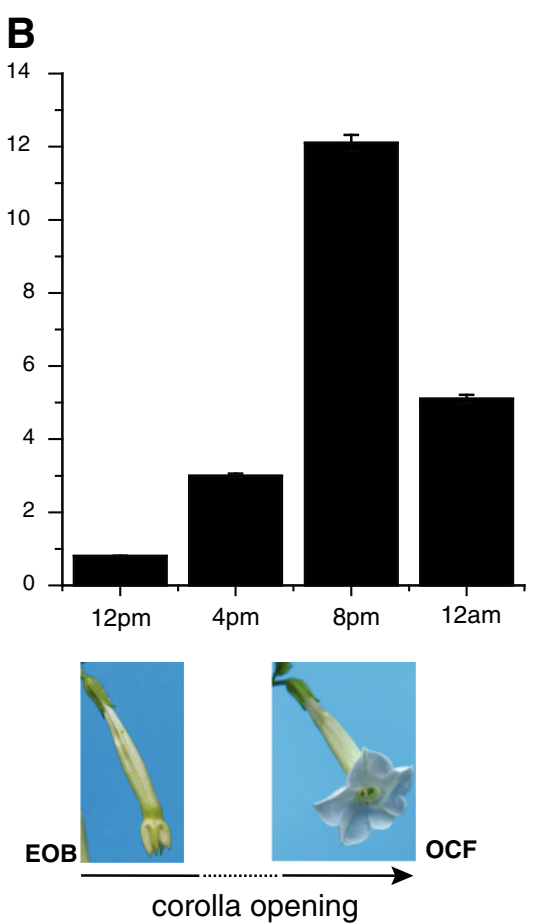

Figure 1 Analysis of HD20 transcript levels during flower development. Total RNA was extracted from both, flowers at different developmental stages and corollas of WT N. attenuata plants. Total RNA was reversed transcribed and the HD20 mRNA levels were quantified by qPCR. Levels are expressed as relative units to the levels of the reference EF1A mRNA as calculated by the $\Delta C T$ method ( $n=3$, bars denote \pm SE). (A) HD20 transcript levels were quantified in closed buds (CB), opened buds (OB), elongating opened buds (EOB), opened corolla flowers (OCF), and isolated corollas. Samples were harvested at 5 pm. (B) HD20 transcript levels were quantified in isolated corollas at different times during corolla opening.

\section{HD20 positively regulates benzyl acetone emission from corollas}

Consistent with our previous observations [27], the emission of BA from corollas of ir-hd20 plants was reduced by $60 \%$ to $70 \%$ compared to corollas of WT plants (Figure 3a; univariate ANOVA, $F_{2,37}=5.65 P<0.01$ followed by a LSD post-hoc test $P<0.01$ for both lines versus WT). In this case, the emitted BA was trapped from individual flowers from $3 \mathrm{pm}$ to $9 \mathrm{am}$ (18 $\mathrm{h}$ trapping period). BA was the only volatile detected by GC-MS (gas chromatography-mass spectrometry) from flower headspace samples that showed differential accumulation in ir- $h d 20$ samples compared to WT. A second trapping period was also carried out during the second day after the corolla opening (from $3 \mathrm{pm}$ to $9 \mathrm{am} ; 18 \mathrm{~h}$ trapping period). In this case, the levels of emitted BA were similar between WT and ir-hd20 plants and were less than $5 \%$ of the BA levels emitted during the first night (Figure 3a). The analysis of BA levels retained in the corolla after the first night showed that these levels were 1,000 times lower than the amounts of emitted BA (Figure 3a). Analysis of BA emission in real time with a $\mathrm{zNOSE}^{\mathrm{TM}}$ (Figure 3b; univariate ANOVA, 10 pm: $F_{2,12}=4.18 P<0.05,12$ am: $F_{2,12}=4.20 P<0.05$ followed by a LSD post-hoc test $P<0.05$ for both lines versus WT) showed that the amount of BA released by corollas of ir$h d 20$ plants was significantly reduced compared to those of WT plants only after $8 \mathrm{pm}$.

\section{Changes in ABA levels correlate with changes in BA emission during corolla opening}

To begin to investigate the mechanisms underlying the reduced emission of BA from corollas of ir-hd20 plants, we first profiled the accumulation of phytohormones in this tissue during the period of corolla opening. The levels of jasmonic acid (JA) and salicylic acid (SA) transiently increased during corolla opening to reach maximum levels at $8 \mathrm{pm}$ and to decrease afterwards (Additional file 3). The levels of JA-Ile in corollas were on average 4 to 10 fold higher than the levels of JA (depending on the time) and they fluctuated on average between 0.5 and $1 \mu \mathrm{gFW}^{-1}$ (Additional file 3). The levels of JA, JA-Ile and SA were however not significantly different between WT and ir- $h d 20$ plants. In contrast, the levels of ABA differed between these two genotypes. In corollas of WT plants, the level of ABA started to increase at $12 \mathrm{pm}$ and reached $20 \mu \mathrm{gFW}^{-1}$ at $4 \mathrm{pm}$ (Figure $4 \mathrm{a}$ ). The levels remained approximately constant until $8 \mathrm{pm}$ and then started to decrease to reach $10 \mu \mathrm{g} \mathrm{gFW}^{-1}$ at $12 \mathrm{am}$ (Figure 4a). This oscillation in ABA levels was not observed 
A
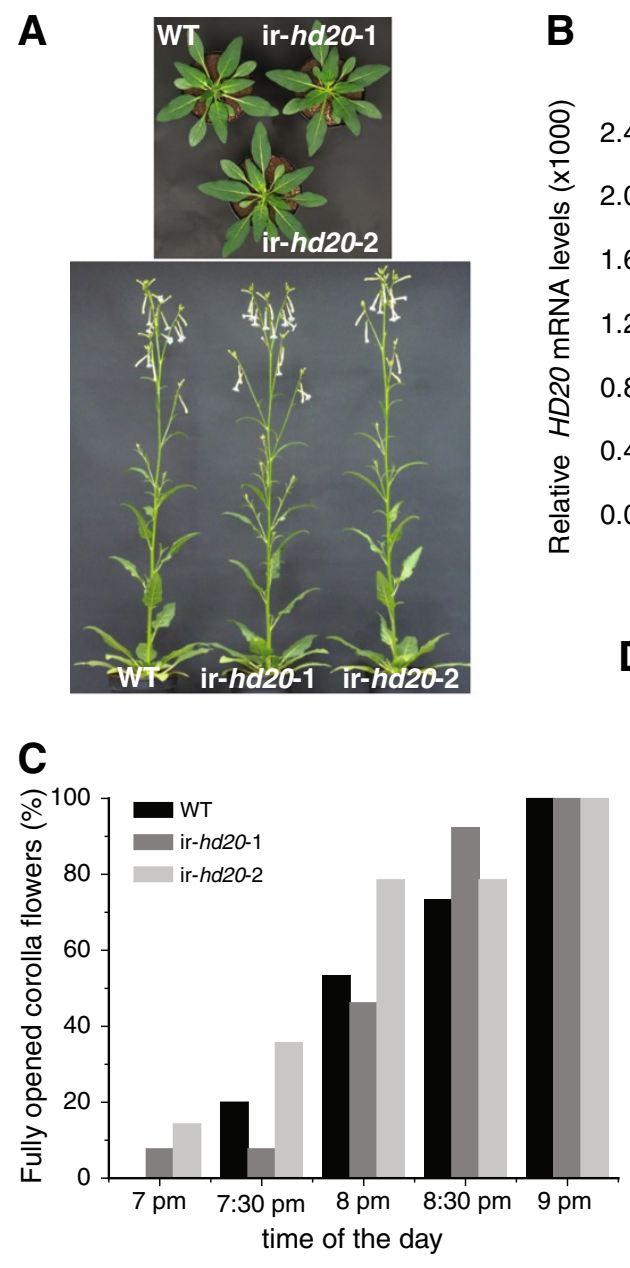

B
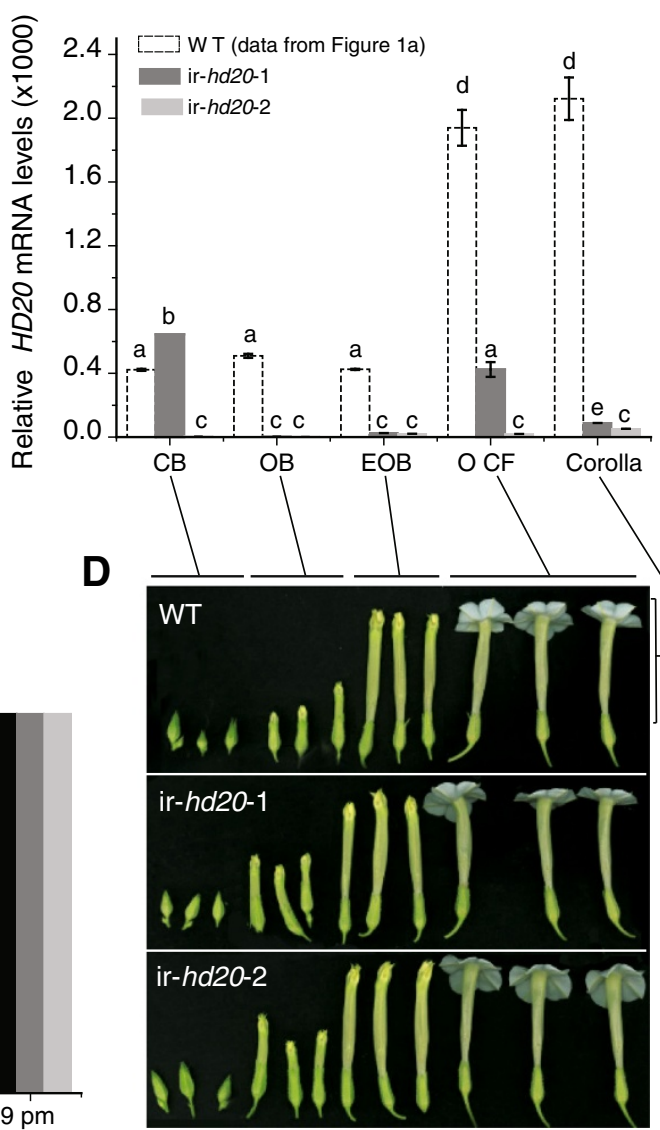

Figure 2 Morphology and development of ir-hd20 N. attenuata plants. (A) Morphology of ir-hd20 and WT plants in early rosette and elongated stages. (B) Analysis of HD20 mRNA expression in flowers of ir-hd20 and WT plants at different developmental stages. The data for WT plants is identical as for Figure 1a and was included only for comparison. Samples were harvested at 5 pm. HD20 transcript levels were quantified as detailed in legend of Figure 1. Different letters denote significant differences; univariate ANOVA, $F_{14,44}=26.64 P<0.001$ followed by a LSD posthoc test $P<0.01$ (different letters denote significant differences). (C) Kinetic of flower opening in ir-hd20 and WT plants ( $n=10)$. (D) Morphology of flowers from ir-hd20 and WT plants at different developmental stages (CB: closed bud; OB: opened bud; EOB: elongating opened bud; OCF: opened corolla flower).

in corollas of ir-hd20 plants, where ABA levels remained approximately constant at 20 to $24 \mu \mathrm{g} \mathrm{gFW}^{-1}$ during the time of corolla opening (Figure 4a; univariate ANOVA, 12 pm: $F_{2,11}=8.25 P<0.01$ followed by a LSD post-hoc test $P<0.01 ; 12$ am: $F_{2,11}=5.39 P<0.05$ followed by a LSD post-hoc test $P<0.05$ for both lines versus WT). Finally, the levels of ethylene emitted by the corolla were also quantified between 4 to $8: 30 \mathrm{pm}$ and 6 to $10: 30 \mathrm{pm}$ and they were similar between WT and ir- $h d 20$ plants (Additional file 3).

To investigate whether the changes in ABA levels quantified in corollas of ir-hd20 plants were specific to this tissue and not the effect of altered ABA accumulation in the whole flower during development, phytohormone levels were quantified in whole flowers (corollas plus pistils, stamens, ovaries and nectaries) at progressive developmental stages. The results showed that accumulation of ABA in whole flowers was similar between WT and ir- $h d 20$ plants (Additional file 4) and hence the changes in ABA accumulation in corollas of ir- $h d 20$ plants do not result from a general alteration of whole flower ABA levels.

To further investigate this association, $N$. attenuata WT and ir- $h d 20$ plants were grown under salt stress, a condition that maintained ABA in corollas at levels lower than $20 \mu \mathrm{g}$ $\mathrm{gFW}^{-1}$ and almost completely abrogated the developmental fluctuation of this phytohormone in corollas of WT plants (Figure 4a). The salt stress did not affect BA emission from corollas of WT plants (compared to control growth conditions; Figure $4 \mathrm{~b}$ ). However, salt stress conditions recovered BA emission in ir- $h d 20$ plants and the levels of emitted BA 

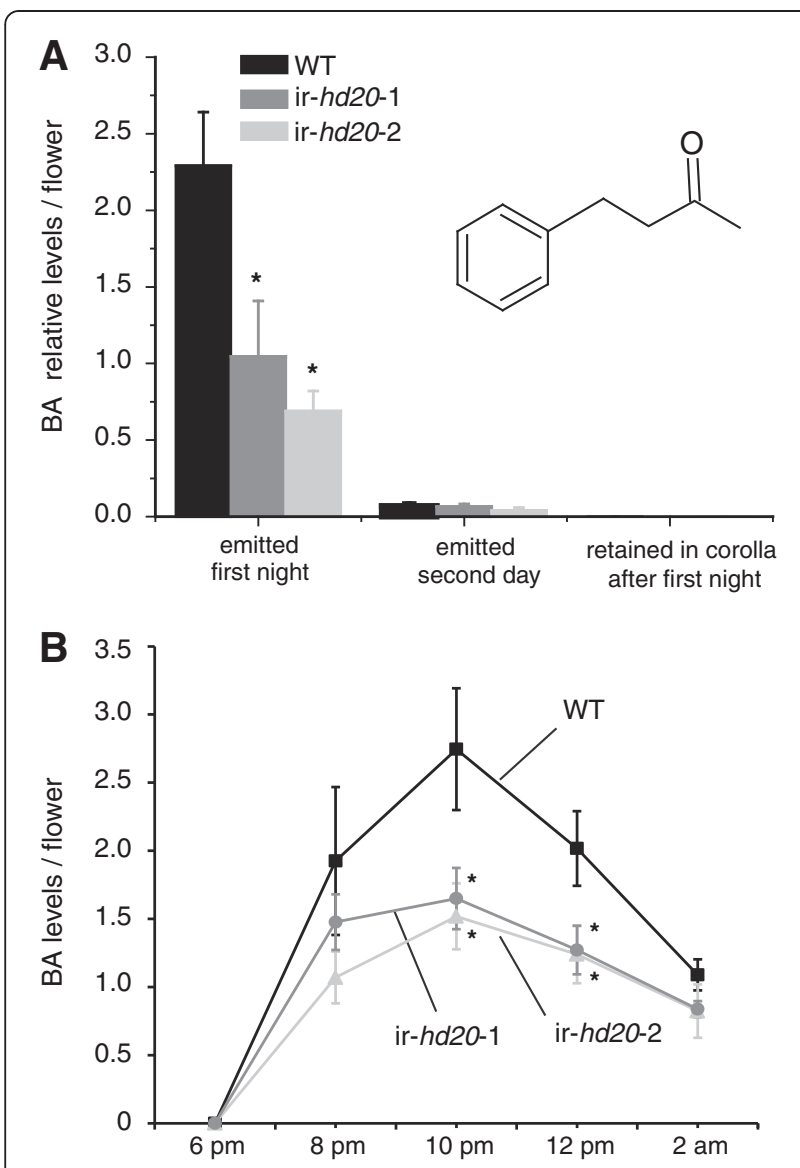

Figure 3 Analysis of BA produced in corollas of ir-hd20 and WT plants. (A) The BA emitted from individual night flowers was trapped from $3 \mathrm{pm}$ to $9 \mathrm{am}$ and analyzed by GC-MS. The BA levels are expressed as the normalized peak areas to tetralin (internal standard; IS). Emitted BA was analyzed from both flowers that just opened their corollas (emitted first night) and from the same flowers but the day after (emitted second day). BA was also extracted from corolla tissue after the first night (retained in corollas after the first night). Asterisks denote significant differences; univariate ANOVA, $F_{2,37}=5.65 P<0.01$ followed by a LSD post-hoc test $P<0.01$ for both lines versus WT; $n>10$; bars denote \pm SE. (B) Kinetic of BA emission from flowers at different times during corolla opening. Emitted BA was analyzed in real time with a $\mathrm{ZNOSE}^{\mathrm{TM}}$. Asteriks denote significant differences; univariate ANOVA, $10 \mathrm{pm}: F_{2,12}=4.18 P<0.05,12 \mathrm{am}$ : $F_{2,12}=4.20 P<0.05$ followed by a LSD post-hoc test $P<0.05$ for both lines versus WT; $n>10$; bars denote $\pm \mathrm{SE}$ ).

were similar to those in WT plants (Figure 4b). From these results, we hypothesized that high levels of ABA in corollas (i.e. $20 \mu \mathrm{g} \mathrm{gFW}^{-1}$ or more) have a negative effect on BA emission and that the developmentally controlled 2-fold reduction in ABA levels in corollas of WT plants contributes to the emission of this volatile. To test this hypothesis, exogenous ABA was supplied to flowers of WT plants and the level of BA emitted from the corollas was quantified. Two different approaches were used: (1) flowers attached to the plant were sprayed with $1 \mathrm{~mL}$ of a solution containing
$1 \mu \mathrm{g} \mathrm{mL} \mathrm{m}^{-1} \mathrm{ABA}$ at $8 \mathrm{pm}$, and (2) clipped flowers were placed in scintillation vials and continuously fed through the pedicle with $100 \mu \mathrm{L}$ of a solution containing $1 \mu \mathrm{g} \mathrm{mL}{ }^{-1}$ ABA. The levels of ABA used in these experiments were in the range of endogenous ABA levels quantified in corollas (for spraying, we estimated a $90 \%$ loss of solution by measuring the run off). In the first approach, the emitted BA was trapped from $3 \mathrm{pm}$ to 9 am $(18 \mathrm{~h})$ and analyzed by GC-MS (Figure 4c). In the second approach, the emitted BA was analyzed every two hours starting at $6 \mathrm{pm}$ with a $\mathrm{zNOSE}^{\mathrm{TM}}$ (Figure $4 \mathrm{~d}$ ). Corollas from WT plants sprayed with ABA emitted 30\% less BA than control-treated flowers during the night (Figure 4c). Consistently, continuously pedicle-fed flowers emitted 25 to $50 \%$ lower levels of BA at 12 am and 2 am compared to control-treated flowers (Figure 4d).

In summary, these experiments indicated that ABA levels in corollas contributed either directly or indirectly to the release of $\mathrm{BA}$. The negative effect of $\mathrm{ABA}$ on $\mathrm{BA}$ emission was however only significant after $10 \mathrm{pm}$, suggesting that the developmentally controlled reduction in ABA levels in corollas of WT plants contributes to the late phase of $\mathrm{BA}$ release. This conclusion is consistent with the reduction in ABA levels after $8 \mathrm{pm}$ in corollas of WT plants (Figure 4a).

\section{Reduced levels of HD20 in corollas have strong effects on gene expression}

To study the effect of HD2O on the expression of genes in opening corollas, a $N$. attenuata Agilent custom-array containing 43,533 probes was used [28,29]. RNA was isolated from corollas of WT and ir- $h d 20$ plants at $12 \mathrm{pm}$ (6 $\mathrm{h}$ before the start of BA emission) and used for microarray hybridization (see Materials and Methods for a detailed description of the method and data analysis). The results of the analysis showed that 215 genes were down- and 59 up-regulated (ir-hd20 vs. WT; $0.6>$ FC (fold change) $>1.9$ ) significantly (q-value $<0.048$; FDR $5.2 \%)$ in corollas of ir-hd20 plants compared to WT (Additional file 5 and Figure $5 \mathrm{a}$ ).

The genes affected in their expression were first categorized based on gene ontology (GO; process and function). Based on these two categories, between 44 and $37 \%$ of the annotated genes were involved in metabolic processes, respectively (Figure 5b, bars labeled with "M"). Analysis of GO categories (EC; Additional file 5), revealed that the most prevalent changes in gene expression occurred in enzymes involved in metabolic processes associated to aromatic compounds (Table 1). More specifically, genes involved in the metabolism of the aromatic amino acids tyrosine, tryptophan and phenylalanine (derivatives of the shikimate pathway) and the biosynthesis of phenylpropanoid derivatives were differentially expressed in corollas of ir-hd20 plants 

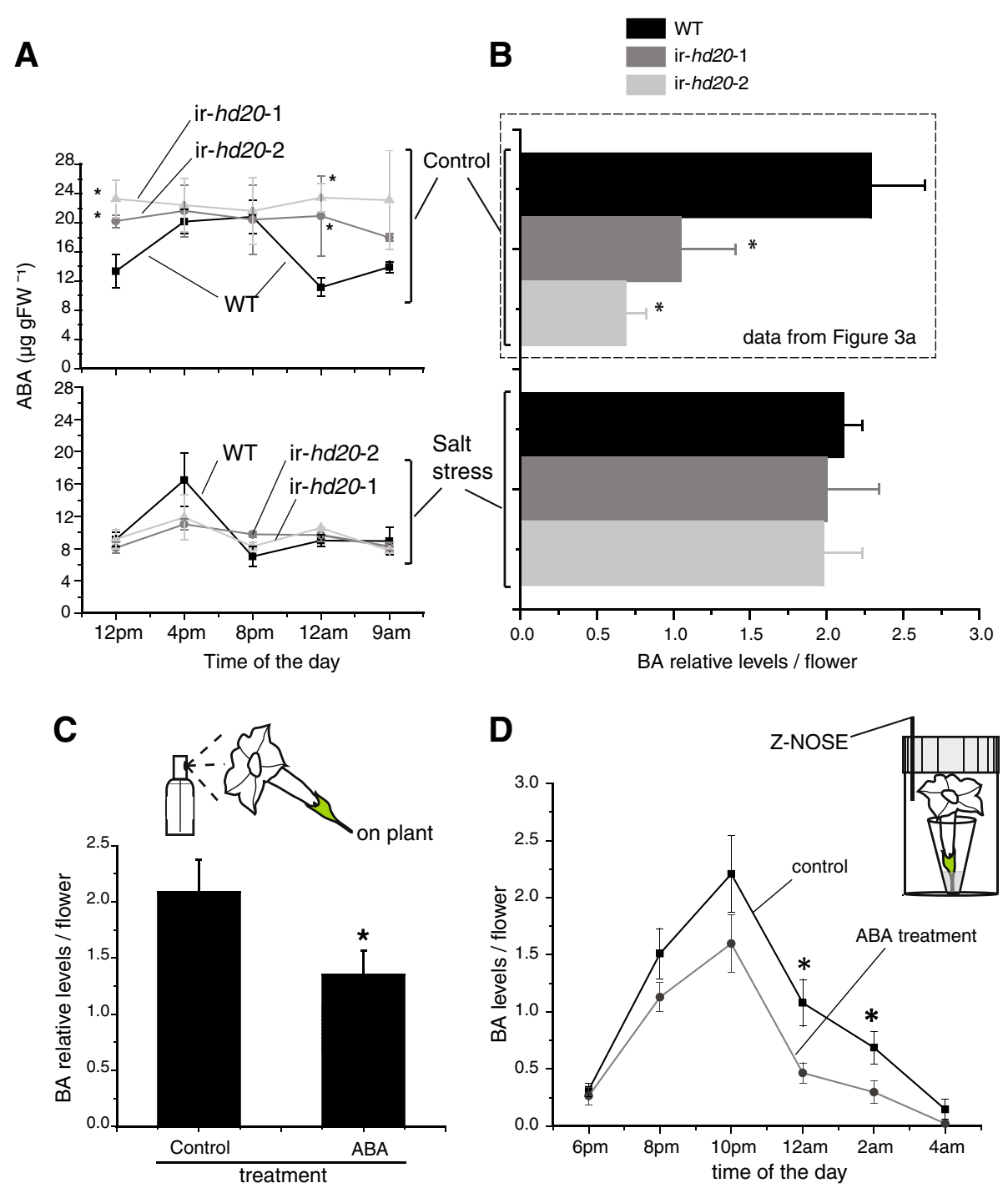

Figure 4 Analysis of ABA levels in corolla and their effect on BA emission. (A) ABA levels were quantified by LC-MS/MS in isolated corollas from flowers of WT and ir-hd20 plants grown either under control or salt stress conditions. (B) BA was trapped from individual flowers from 3 pm to 9 am during the first night of corolla opening. WT and ir-hd20 plants were grown either under control or salt stress conditions (BA levels in control conditions correspond to the data showed in Figure $3 a$ and were included here for comparison). (C) Flowers from WT plants were sprayed either with $1 \mathrm{~mL}$ of solvent or with $1 \mathrm{~mL}$ a solution containing $A B A\left(1 \mu \mathrm{g} \mathrm{mL} \mathrm{L}^{-1}\right)$ at $8 \mathrm{pm}$. BA was trapped from individual flowers from $10 \mathrm{pm}$ to $9 \mathrm{am}$ and analyzed by GC-MS. BA levels are expressed as detailed in legend of Figure 3. (D) Flowers from WT plants were clipped off at the base of the pedicle and placed inside a microcentrifuge tube containing either $100 \mu \mathrm{L}$ of water or $100 \mu \mathrm{L} \mathrm{ABA}\left(1 \mu \mathrm{g} \mathrm{mL}{ }^{-1}\right)$. Emitted BA was analyzed in real time with a zNOSE ${ }^{\mathrm{TM}}$. Asterisks correspond to $P<0.05$ (Student's $t$-test; ir-hd20 vs. WT levels; $n=6$; bars denote \pm SE).

compared to WT (Table 1$)$. A putative $L$-amino-acid oxidase (EC:1.4.3.2) involved in the metabolism of tyrosine, tryptophan and phenylalanine and an unspecific monooxygenase (EC:1.14.14.1) involved in the metabolism of tryptophan were down-regulated in corollas of ir- $h d 20$ plants while the transcript levels of the rest of the genes involved in aromatic compound metabolism were upregulated (Table 1). The second largest group of metabolic genes affected in their expression in corollas of ir- $h d 20$ plants was associated with carbohydrate metabolism
(Table 1). In this case, several genes involved in sugar metabolism were down-regulated while two genes involved in cell wall biosynthesis were up-regulated. Core genes of the fatty acid and lipid biosynthesis pathways were also affected, and in this case they were up-regulated in corollas of ir$h d 20$ plants (Table 1). Together, the changes in the expression of genes involved in aromatic compound metabolism and in sugar and lipid metabolism suggested that HD20 participates either directly or indirectly in the control of primary and secondary metabolism in corollas of $N$. attenuata. 


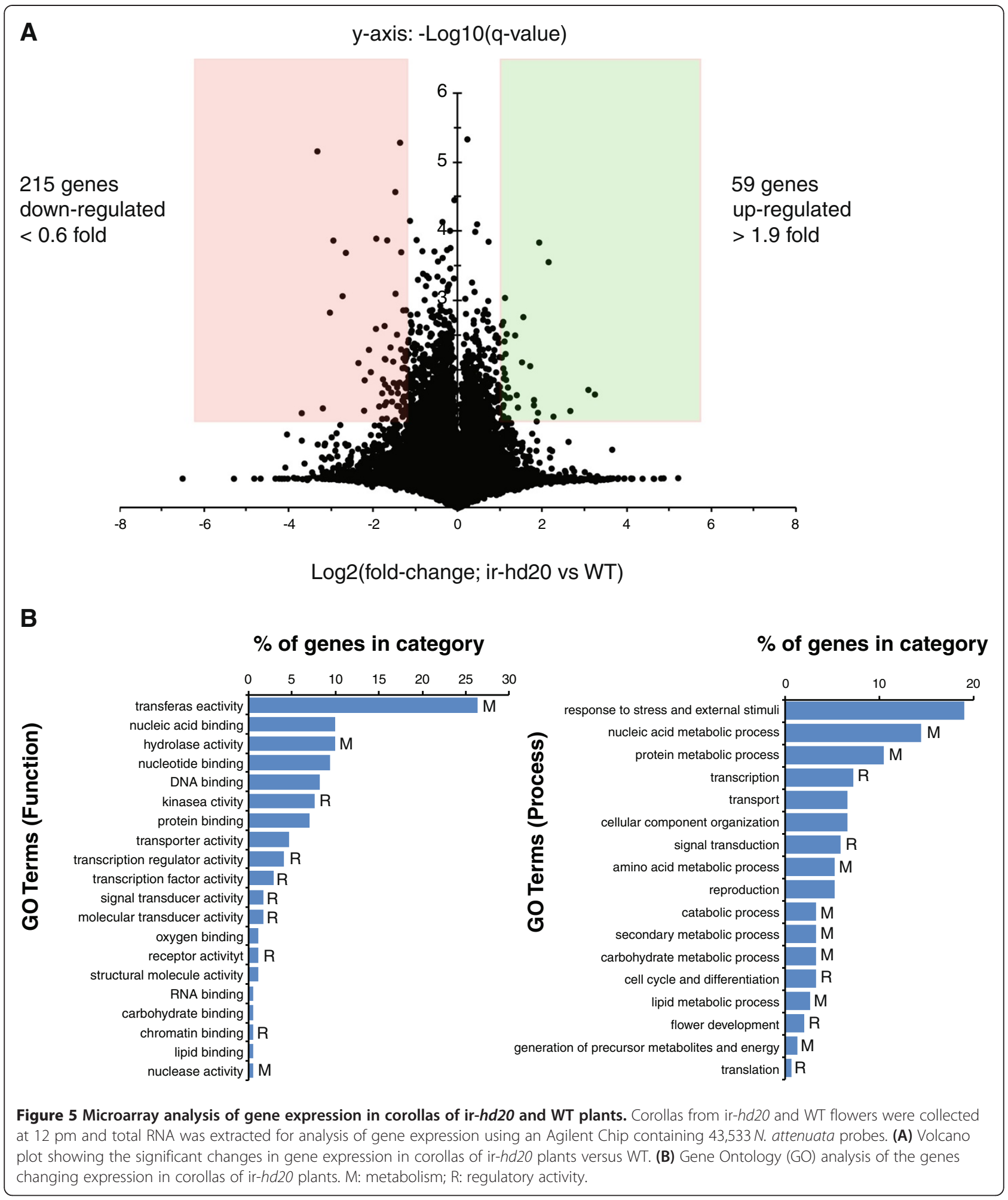

GO categorization also showed that $20 \%$ of the genes affected in their expression were involved in regulatory processes (Figure 5b, bars labeled with "R"). Among these genes, the most prevalent group was associated to the regulation of gene expression and it included two WRKY transcription factors and two chromatin-remodeling factors (Table 2). The expression of several genes with protein kinase activity (including receptors such as ETR1) was also affected (Table 2). Among the down-regulated receptor proteins was CORONATINE INSENSITIVE 1 (COI1), 
Table 1 List of selected genes involved in biochemical pathways

\begin{tabular}{|c|c|c|c|c|}
\hline Metabolic pathway & Gene function & Gene ID & $\mathrm{FC}^{*}$ & q-value \\
\hline \multicolumn{5}{|l|}{ Aromatic compounds } \\
\hline \multirow[t]{3}{*}{ Tryptophan metabolism } & EC:1.14.14.1 - unspecific monooxygenase & Na_14909 & 0.6 & 0.0241 \\
\hline & EC:1.4.3.2 - L-amino-acid oxidase & Na_16251 & 0.6 & 0.0447 \\
\hline & EC:4.1.1.28 - aromatic-L-amino-acid decarboxylase & Na_32067 & 2.1 & 0.0080 \\
\hline \multirow[t]{3}{*}{ Tyrosine metabolism } & EC:1.4.3.2 - L-amino-acid oxidase & $\mathrm{Na} 16251$ & 0.6 & 0.0447 \\
\hline & EC:4.1.1.28 - aromatic-L-amino-acid decarboxylase & Na_32067 & 2.1 & 0.0080 \\
\hline & EC:4.1.1.25 - tyrosine decarboxylase & Na_32067 & 2.1 & 0.0080 \\
\hline \multirow[t]{2}{*}{ Phenylalanine metabolism } & EC:1.4.3.2 - L-amino-acid oxidase & Na_16251 & 0.6 & 0.0447 \\
\hline & EC:4.1.1.28 - aromatic-L-amino-acid decarboxylase & Na_32067 & 2.1 & 0.0080 \\
\hline Biosynthesis of & EC:1.1.1.219 - dihydroflavonol 4-reductase & Na_06408 & 2.1 & 0.0058 \\
\hline \multirow[t]{2}{*}{ phenylpropanoid derivatives } & EC:2.3.1.74 - chalcone synthase & Na_02855 & 2.1 & 0.0241 \\
\hline & EC:1.14.11.19 - leucocyanidin oxygenase & $\mathrm{Na} 12114$ & 2.2 & 0.0019 \\
\hline \multicolumn{5}{|l|}{ Carbohydrate metabolism } \\
\hline Starch and sucrose & EC:2.4.1.21 - starch synthase & Na_41693 & 0.3 & 0.0223 \\
\hline \multirow[t]{3}{*}{ metabolism } & EC:2.7.1.4 - fructokinase & $\mathrm{Na} 26865$ & 0.5 & 0.0226 \\
\hline & EC:2.4.1.15 - alpha,alpha-trehalose-phosphate synthase (UDP-forming) & Na_27299 & 0.5 & 0.0266 \\
\hline & EC:3.1.3.12 - trehalose-phosphatase & Na_27299 & 0.5 & 0.0266 \\
\hline \multirow[t]{2}{*}{ Cell wall biosynthesis } & EC:2.4.1.12 - cellulose synthase (UDP-forming) & Na_10287 & 1.9 & 0.0147 \\
\hline & EC:2.4.1.12 - cellulose synthase (UDP-forming) & $\mathrm{Na} 28221$ & 2.0 & 0.0260 \\
\hline \multicolumn{5}{|l|}{ Lipid metabolism } \\
\hline \multirow[t]{2}{*}{ Glycerophospholipid metabolism } & EC:2.1.1.103 - phosphoethanolamine N-methyltransferase & Na_18104 & 1.9 & 0.0299 \\
\hline & EC:3.1.4.46 - glycerophosphodiester phosphodiesterase & Na_34149 & 2.4 & 0.0117 \\
\hline Fatty acid biosynthesis & EC:2.3.1.86 - fatty-acyl-CoA synthase & $\mathrm{Na} 20395$ & 1.9 & 0.0351 \\
\hline
\end{tabular}

* FC: fold-change (ir-hd20 vs. WT).

previously associated with developmental processes in flowers [30-32].

To further assess the changes in gene expression in corollas of ir- $h d 20$ plants, the kinetics of expression of a selected group of genes identified by microarray analysis were evaluated by qPCR during the time of corolla opening (Figure 6a). Three of the selected genes are involved in aromatic compound metabolism, namely Chalcone Synthase 1 (CHAL1), S-adenosyl-L-methionine:Benzoic acid/Salicylic acid Carboxyl Methyltransferase (SAMT/ $B A M T$ ) and Dihydroflavonol 4-Reductase (DFR)(Table 1). CHAL1 has been previously associated with BA production in $N$. attenuata plants [2]. The accumulation of these three transcripts was induced in corollas of WT plants during the time of corolla opening to reach maximum levels between 4 and 8 pm (Figure 6b,c,d). Consistent with the microarray data, the levels of CHAL1, $S A M T / B A M T$ and DFR mRNAs were increased in corollas of ir- $h d 20$ plants compared to WT (Figure 6b,c,d; univariate ANOVA, $F_{2,8}$ and $P<0.05$ followed by a LSD post-hoc test $P<0.05$ for both lines versus WT).

The expression of two regulatory genes was also analyzed, namely Casein Kinase (CK; the most strongly down-regulated gene; Additional file 5 and Table 2) and
Protein Phosphatase $2 \mathrm{C}(\mathrm{PP} 2 \mathrm{C})$. The expression of $C K$ was induced several fold during corolla opening in WT plants and reached a maximum at $8 \mathrm{pm}$ (Figure 6e). In contrast, in corollas of ir- $h d 20$ plants, the levels of this transcript remained largely non-induced (Figure 6e; univariate ANOVA, $F_{2,8}$ and $P<0.05$ followed by a LSD post-hoc test $P<0.05$ for both lines versus WT). Finally, the levels of $P P 2 C$ mRNA were induced by 2 -fold during corolla opening in WT plants and the levels remained approximately 2 -fold lower in corollas of ir- $h d 20$ plants (Figure 6f; univariate ANOVA, $F_{2,8}$ and $P<0.05$ followed by a LSD post-hoc test $P<0.05$ for both lines versus WT).

\section{Discussion}

\section{Changes in ABA levels in opening corollas are associated} with $B A$ emission

The expression of $H D 20$ in flowers and corollas was consistent with the participation of this transcription factor in the process of BA emission. The levels of HD2O mRNA were highest in corollas and they increased during the time of corolla opening (Figure 1). The timing of induction correlated with the timing of BA emission which starts in the afternoon and peaks between 9 and 
Table 2 List of selected genes involved in regulatory processes

\begin{tabular}{|c|c|c|c|}
\hline Gene description & $\mathrm{FC}^{*}$ & q-value & Gene ID \\
\hline \multicolumn{4}{|l|}{ Gene expression } \\
\hline $\begin{array}{l}\text { GBF S pro-rich region-interacting } \\
\text { factor } 1\end{array}$ & 0.3 & 0.0030 & $\mathrm{Na} 05287$ \\
\hline WRKY transcription factor & 0.3 & 0.0120 & $\mathrm{Na}$ _30800 \\
\hline $\begin{array}{l}\text { Fungal-specific transcription factor } \\
\text { domain protein }\end{array}$ & 0.4 & 0.0140 & $\mathrm{Na} 31163$ \\
\hline $\begin{array}{l}\text { Histone acetyltransferase complex } \\
\text { component }\end{array}$ & 0.4 & 0.0009 & $\mathrm{Na}$ \\
\hline RWP-RK domain-containing protein & 0.5 & 0.0479 & $\mathrm{Na} 20983$ \\
\hline $\begin{array}{l}\text { Chromatin remodeling complex } \\
\text { subunit }\end{array}$ & 0.5 & 0.0395 & Na_16219 \\
\hline WRKY transcription factor 29 & 0.5 & 0.0420 & $\mathrm{Na} 36997$ \\
\hline RNA polymerase sigma factor & 0.6 & 0.0222 & $\mathrm{Na}$ \\
\hline $\begin{array}{l}\text { Paired amphipathic helix protein } \\
\text { SIN3-like } 2\end{array}$ & 0.6 & 0.0415 & $\mathrm{Na}$ _13033 \\
\hline GRAS transcription factor & 4.8 & 0.0360 & $\mathrm{Na}$ _40201 \\
\hline
\end{tabular}

\section{Protein kinases and}

phosphatases

\begin{tabular}{llll}
\hline Casein kinase & 0.1 & 0.0051 & $\mathrm{Na}$ _35212 \\
\hline Ser-Thr protein phosphatase & 0.3 & 0.0158 & $\mathrm{Na}$ _21633 \\
\hline Protein phosphatase 2C & 0.6 & 0.0436 & $\mathrm{Na}$ _30444 \\
\hline TCTR2 protein kinase & 0.4 & 0.0121 & $\mathrm{Na}$ _36065
\end{tabular}

\section{Receptor activity}

\begin{tabular}{llll}
\hline Ethylene receptor 1 (ETR1) & 0.4 & 0.0144 & $\mathrm{Na} 24848$ \\
\hline S-locus lectin protein kinase & 0.5 & 0.0147 & $\mathrm{Na}$ _14354 \\
\hline Coronatine insensitive 1 (COI1) & 0.6 & 0.0283 & $\mathrm{Na}$ _04958 \\
\hline LRR receptor-like kinase & 0.6 & 0.0388 & $\mathrm{Na}$ _29192
\end{tabular}

Other regulatory activities

circadian clock-associated FKF1

* FC: fold-change (ir-hd20 vs. WT).

$10 \mathrm{pm}$ (Figure 3b). Several HD-Zip type-I transcription factors in different plants species are either highly expressed in flowers or have been associated with mechanisms regulating organ development. For example, LeHB-1 from tomato (Solanum lycopersicum) regulates floral organogenesis [24] and the Arabidopsis HB12, $H B 7, H B 6$ and $H B 1$ are highly expressed in flowers $[19,20]$. Based on phylogenetic analysis, the Arabidopsis $H B 12$ and $H B 7$ genes group together with HD2O in subgroup Ic $[27,33]$.

Consistent with our previous study in which the expression of $H D 20$ was reduced by VIGS in N. attenuata plants [27], plants with stably reduced expression of HD20 were also reduced in the levels of BA released from corollas (Figure 3). Analysis of BA emission during the first and second nights showed that flowers from ir$h d 20$ plants produced less BA with no delay in its emission (Figure 3a). Moreover, analysis of the dynamics of BA emission showed that corollas of ir-hd20 plants released significantly smaller BA amounts after $8 \mathrm{pm}$, indicating that reduced $H D 20$ expression affected primarily the late phase of BA emission (Figure 3b).

In WT plants, HD2O mRNA levels were detected throughout the time of corolla opening (i.e. from $12 \mathrm{pm}$ to $12 \mathrm{am}$ ) and peaked at $8 \mathrm{pm}$ (Figure 1b). ABA levels increased in corollas of WT plants from $12 \mathrm{pm}$ to $4 \mathrm{pm}$ and decrease from $8 \mathrm{pm}$ to 9 am (Figure 4a). In corollas of ir- $h d 20$ plants, this fluctuation in ABA levels was not observed (Figure 4a) however when ABA levels in corollas were reduced by salt stress treatment, the emission of BA was recovered to WT levels (Figure $4 \mathrm{~b}$ ). Moreover, feeding experiments with exogenous ABA decreased BA emission at the late phase of the process (i.e. after $10 \mathrm{pm}$; Figures $4 \mathrm{c}$ and d). Based on these observations, we concluded that there was a direct association between the decline of ABA levels in corolla and the late phase of BA emission. Thus, the results suggested that HD20 contributes partially to $\mathrm{BA}$ emission by directly or indirectly affecting ABA levels. How HD20 affects ABA levels in corollas is at present unknown. Genome-wide gene expression analysis performed with corolla tissue harvested at $12 \mathrm{pm}$ ( $6 \mathrm{~h}$ before the start of BA emission) did not detect genes involved in ABA biosynthesis as differentially expressed. At present, however, it is unknown how the corolla controls ABA levels, this could be the result of de novo biosynthesis, import from adjacent tissue, metabolism (i.e. turnover) or a combination of these processes. Although at $12 \mathrm{pm}, 2$-fold higher levels of ABA were detected in corollas of ir-hd20 plants compared to WT (Figure 4a), only one gene homolog to Zea mays SnRK2 (known to be regulated by ABA [34]) was identified as differentially expressed (supplementary file 5). Litthe is known about the regulation of gene expression by $\mathrm{ABA}$ in corollas and $\mathrm{ABA}$ is known to affect multiple cellular processes (e.g., opening of ion channels, protein phosphorylation) in addition to gene expression [35]. Moreover, the direct target genes of HD20 are thus far unknown in $N$. attenuata. The mechanisms underlying the reduction in BA emission mediated by HD20 and ABA are probably very complex and additional experimentation will be required to disentangle this complexity.

\section{Changes in metabolic gene expression induced by HD20 are associated with BA emission}

Chalcone Synthase 1 (CHAL1) is so far the only gene that has been involved in the biosynthesis of BA in $N$. attenuata plants [2]. In other words, the biosynthesis pathway for this floral volatile is largely unknown. Chalcone synthases catalyze the first step of flavonoid biosynthesis using malonyl-coenzyme A (CoA) and 4coumaroyl-CoA to produce naringenin chalcone [36]. 4coumaroyl-CoA is supplied by the phenylpropanoid 


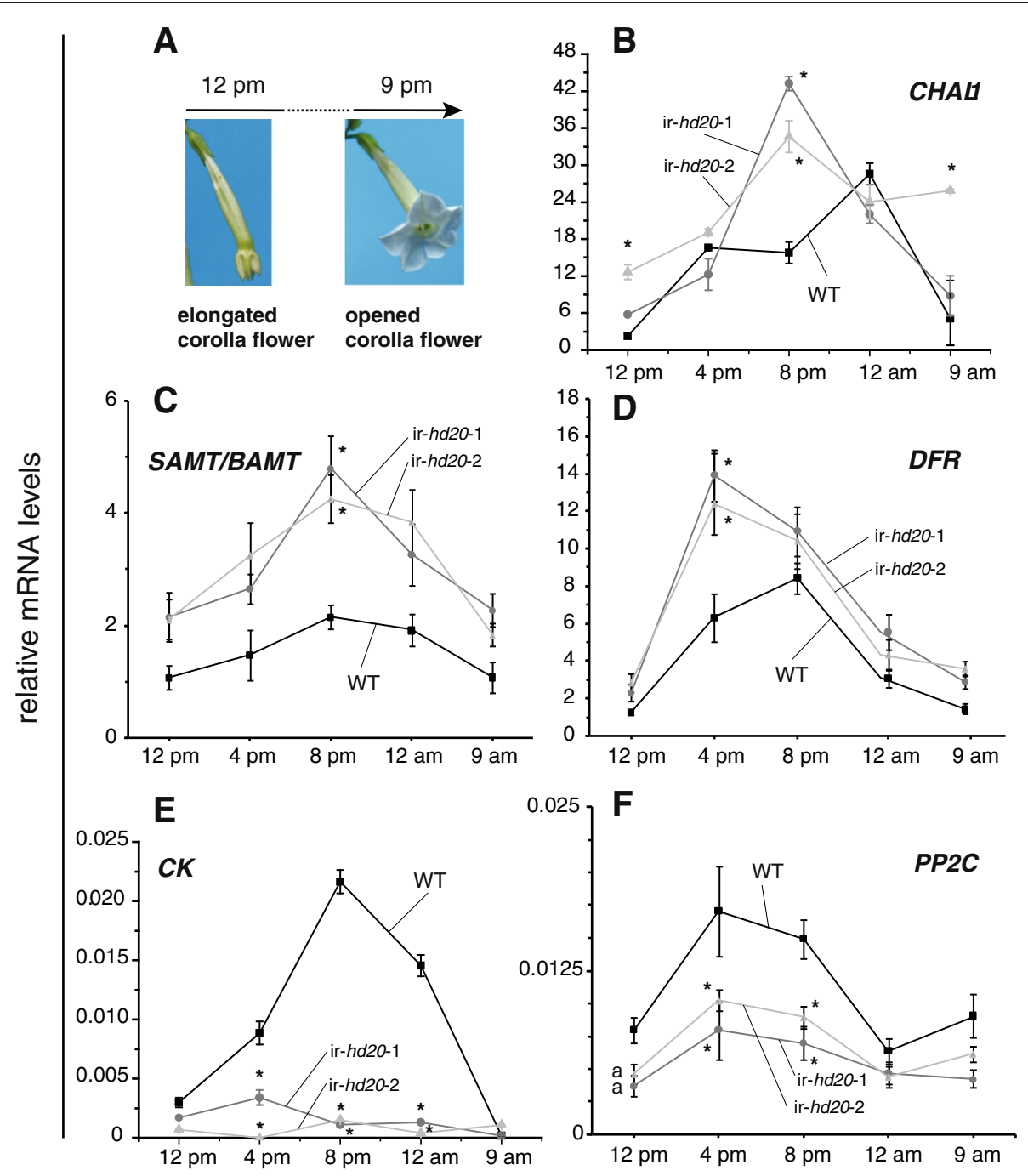

Figure 6 Analysis of gene expression during corolla opening by qPCR. Total RNA was extracted from isolated corollas of ir-hd20 and WT plants at different times during corolla opening. Total RNA was reversed transcribed and the mRNA levels for the different genes were quantified by $\mathrm{qPCR}$. Transcript levels are expressed as relative units to the levels of the reference EFIA mRNA as calculated by the $\triangle C T$ method. Asterisks denote significant differences; univariate ANOVA $F_{2,8}$ and $P<0.05$ followed by a LSD post-hoc test $P<0.05$ for both lines versus WT; $n=3$; bars denote \pm SE. (A) Schematic representation of corolla opening. (B) CHAL1 mRNA levels. (C) SAMT/BAMT mRNA levels. (D) DFR mRNA levels. (E) CK mRNA levels. (F) PP2C mRNA levels.

pathway which utilizes phenylalanine produced by the shikimate pathway in the plastid. Tyrosine and tryptophan are also produced by this biosynthesis pathway. Naringenin chalcone can be further modified to form flavonoids and dihydroflavonol 4-reductase (DFR) is the first committed enzyme of anthocyanin biosynthesis in the flavonoid pathway [36]. Benzoids are also derived from the phenylpropanoid or shikimate pathways and enzymes such as S-adenosyl-L-methionine:benzoic acid/ Salicylic acid carboxyl methyltransferase (BAMT/SAMT) synthesize methyl esters (e.g., methyl benzoate and methyl salicylate) which are constituents of floral scents of many plant species [1]. In Petunia hybrida, where benzenoids determine the floral scent, three transcription factors from the R2R3-type MYB family have been identified that regulate floral scent production. ODORANT1 (ODO1), EMISSION OF BENZENOIDS II (EOBII) and PhMYB4 affect the expression of several biosynthetic floral scent-related genes encoding enzymes from the phenylpropanoid and shikimate pathways $[5,6,37]$.

The analysis of changes in gene expression in opening corollas of ir- $h d 20$ plants showed that the most prevalent group of genes with altered expression corresponded to those involved in aromatic compound metabolism and derivatives of the phenylpropanoid biosynthesis pathway. Importantly, no candidate genes involved in BA 
biosynthesis could be identified, however, as the genes involved in this process are largely unknown and a large fraction of the genes differentially expressed in corollas of ir- $h d 20$ are of undescribed function (supplementary file 5), it is plausible that among these genes some involved in BA biosynthesis are present.

The induction of expression of genes involved in aromatic compound metabolism and derivatives of the phenylpropanoid biosynthesis pathway during corolla opening (e.g., CHAL1, SAMT/BAMT, DFR) correlated positively with the emission of BA and with the turning of the corolla limb from green to white. Thus, these observations were consistent with the altered levels of BA production in ir-hd20 plants and suggested that reduced emission of this floral volatile could be the result of altered gene expression in BA biosynthesis genes. Paradoxically, however, in addition to CHAL1, several other genes involved in different steps of aromatic compound biosynthesis or metabolism were upregulated in corollas of ir- $h d 20$ plants (Table 1). Because several genes involved in carbohydrate metabolism were down-regulated in corollas of these plants (Table 1), one plausible explanation for the reduced levels of BA emission in ir- $h d 20$ plants is that reduced carbon fluxes through the shikimate and phenylpropanoid pathways limit BA production. If this is the case, the increased levels of chalcone synthase and other phenylpropanoid biosynthesis genes observed in ir- $h d 20$ plants may reflect a compensatory mechanism for reduced supply of carbon precursors. A detailed study of the metabolic fluxes of the shikimate and phenylpropanoid pathways in ir-hd20 plants will be necessary to test this hypothesis in the future.

In addition to metabolic genes, multiple regulatory factors also showed altered expression in opening corollas of ir- $h d 20$ plants (Table 2). Among the genes in the group of protein kinases and phosphatases were $N$. attenuata homologues of casein kinase and PP2C (Table 2). Casein kinases (CKs) are ubiquitous Ser/Thr kinases that play critical roles in all higher organisms including plants. For example, the Arabidopsis CK2 plays important roles in light signaling, circadian rhythms, hormone responses, cell cycle control and flowering time $[6,38,39]$. The strong down-regulation of casein kinase suggests that this protein kinase may be important in regulating directly or indirectly BA emissions by HD20dependent mechanisms. Interestingly, similar to HB12 and HB7 in Arabidopsis [33], the carboxyl terminus of HD20 presents three putative phosphorylation sites (data not shown), suggesting that phosphorylation/dephosphorylation mechanisms can be important for the regulation of its activity.

In connection with $\mathrm{ABA}$ signaling, a homologue of PP2C was down-regulated approximately two-fold in opening corollas of ir- $h d 20$ plants (Table 2 and Figure $6 f$ ). PPC2s such as ABI1 and ABI2 (Abscisic Acid Insensitive
1 and 2, respectively) are rapidly inactivated upon the binding of ABA to RCARs/PYR1/PYLs [5,40,41]. Inactivation of PP2Cs triggers the activation of SNF1-type kinases that initiate ABA-dependent responses such as the activation of gene expression and the regulation of ion channels $[5,40,41]$. As their names indicate, mutations in $A B I 1$ and $A B I 2$ genes make plants insensitive to ABA [42]. Functional $A B I 1$ and $A B I 2$ alleles are necessary for the induction of the Arabidopsis $H B 12$ and $H B 7$ by ABA [20]. Thus, in addition to the de-regulation of ABA levels in corollas of ir- $h d 20$ plants, sensitivity to this phytohormone could also be affected in corollas of these plants.

Other interesting genes with receptor activity that changed expression in opening corollas of ir- $h d 20$ compared to WT plants were Ethylene receptor 1 (ETR1), Coronatine insensitive 1 (COI1) and the circadian clockassociated Flavin-binding Kelch repeat F-box 1 (FKF1) gene [43]. Ethylene and JA-Ile are important phytohormones for flower development. For example, plants deficient in the expression of COI1 are sterile $[31,32,44]$ and plants expressing a constitutively active allele of ETR1 have delayed corolla senescence [45,46]. Although JA, JA-Ile and ethylene levels were not affected in corollas of ir- $h d 20$ plants, changes in the sensitivity to these phytohormones may also participate in the regulation of BA emission.

\section{Conclusion}

In summary, from the results presented in this study it is clear that multiple metabolic pathways were affected when corollas were reduced in HD2O expression, pointing to the importance of this HD-Zip transcription factor in the regulation of biochemical processes in this tissue. Importantly, reduced expression of HD2O did not cause morphological and developmental changes in flowers, indicating that the main function of this transcription factor in this organ relates to the regulation of metabolism rather than development. The mechanisms underlying the regulation of BA levels by HD20 are clearly complex and they most likely involve a complex network of factors and $\mathrm{ABA}$ signaling. As the sequences of the promoter regions of $N$. attenuata genes become available with the sequencing of this plant's genome, the analysis of the presence of the universally conserved HD-Zip type I binding element (CAAT(A/T)ATTG) $[14,15]$ in genes affected in their expression in ir- $h d 20$ plants will facilitate the discovery of direct target genes of HD20.

\section{Methods}

\section{Plant growth and treatments}

Seeds of $N$. attenuata plants were germinated on agar plates containing Gamborg's B5 medium as previously described 
[47]. Plates were maintained in a growth chamber (Snijders Scientific, Tilburg, Netherlands) at $26^{\circ} \mathrm{C} / 16 \mathrm{~h}$ (155 $\mu \mathrm{mol} \mathrm{s}{ }^{-1} \mathrm{~m}^{-2}$ light), $24^{\circ} \mathrm{C} / 8 \mathrm{~h}$ dark for 10 days. Ten-day old seedlings were transferred to TEKU pots (Pöppelmann GmbH \& Co. KG, Lohne, Germany) with Klasmann plug soil (Klasmann-Deilmann $\mathrm{GmbH}$, Geesten, Germany). After 10 days, seedlings were transferred to soil in $2 \mathrm{~L}$ pots and grown in the glasshouse under high-pressure sodium lamps $\left(200-300 \mu \mathrm{mol} \mathrm{s}^{-1} \mathrm{~m}\right.$ $\left.{ }^{-2}\right)$ with a day/night ratio of $16 \mathrm{~h}\left(26-28^{\circ} \mathrm{C}\right) / 8 \mathrm{~h}\left(22-24^{\circ} \mathrm{C}\right)$ and $45-55 \%$ humidity. Plants were grown in pairs (one WT and one ir-hd20 per 2-L pot).

For salt stress treatments, elongated $N$. attenuata plants were watered daily under a regime of: 2 days with an aqueous solution of $75 \mathrm{mM} \mathrm{NaCl}, 3$ days with $150 \mathrm{mM} \mathrm{NaCl}$ and 4 days with $300 \mathrm{mM} \mathrm{NaCl}$. The plants were left to soak the saline water for $1 \mathrm{~h}$ and the excess of liquid was removed from the trays. The saline water volume was $0.6 \mathrm{~L}$ per tray and 5 plant pots were placed per tray.

For exogenous ABA application, a solution of $1 \mu \mathrm{g} \mathrm{mL}$ ABA in $0.02 \%(\mathrm{v} / \mathrm{v})$ Tween-20/water was sprayed (1 mL per flower) on corollas at $8 \mathrm{pm}$ with a perfume dispenser. The control solution was $0.02 \%(\mathrm{v} / \mathrm{v})$ Tween-20/water. Flowers were immediately enclosed in volatile traps for BA analysis by GC-MS (see below). For pedicle feeding of ABA, individual clipped flowers were placed inside $1.7 \mathrm{~mL}$ microcentrifuge tubes (with their caps removed) containing $100 \mu \mathrm{L}$ of either water or ABA $\left(1 \mu \mathrm{g} \mathrm{mL} \mathrm{m}^{-1}\right)$ dissolved in water. The microcentrifuge tubes carrying the flowers were placed inside glass scintillation vials and the vials were screw-capped. $\mathrm{BA}$ levels were determined with a $\mathrm{zNOSE}^{\mathrm{TM}}$ (Electronic Sensor Technology, Newbury Park, CA) every 2 h (see below), from $6 \mathrm{pm}$ to $4 \mathrm{am}$. These experiments were performed with 6 biological replicates per genotype.

\section{Generation of transgenic ir-hd20 lines}

Transgenic $N$. attenuata (ir-hd20) plants reduced in the expression of $H D 20$ were generated via elongated hypocotyl Agrobacterium-mediated transformation and seedling regeneration as previously described [47]. The binary vector used for plant transformation was pSOL8 [48] engineered to carry a 329 bp fragment of the HD20 mRNA corresponding to nucleotides 529 to 857 (GenBank accession: HM107874) and subcloned in inverted repeat orientation to generate pSOL8HD20. $\mathrm{T}_{1}$ transformed plants were analyzed by quantification of ABA levels (see below) and HD20 mRNA levels (see below) in leaves during water stress (i.e. by withholding irrigation for 5 days) and for T-DNA single insertion by Southern blot hybridization (see below). Segregation analysis of hygromycin resistance in $\mathrm{T}_{2}$ seedlings was performed on agar plates supplemented with hygromycin $\left(0.025 \mathrm{mg} \mathrm{mL}^{-1}\right)$. Two lines, ir-hd20 A-09-408 (ir-hd20 line 1) and A-09-411 (ir-hd20 line 2) had the lowest levels of ABA and HD20 mRNA accumulation at 5 days after withholding water and had a single T-DNA insertion in their genomes. These lines were used for all experiments. Southern blot analysis was performed as previously described [29].

\section{Analysis of phytohormones}

For analysis of JA, JA-Ile, SA and ABA, $0.2 \mathrm{~g}$ of frozen tissue were homogenized to a fine powder in the presence of liquid nitrogen. One $\mathrm{mL}$ of ethylacetate spiked with $200 \mathrm{ng}$ $\left[{ }^{2} \mathrm{H}_{2}\right]$ JA and $40 \mathrm{ng}\left[{ }^{13} \mathrm{C}_{6}\right]$ JA-Ile, $\left[{ }^{2} \mathrm{H}_{6}\right] \mathrm{ABA},\left[{ }^{2} \mathrm{H}_{4}\right] \mathrm{SA}$ was added to the samples and after vortexing the samples were centrifuged for $15 \mathrm{~min}$ at $13,200 \mathrm{~g}\left(4^{\circ} \mathrm{C}\right)$. The upper organic phase was transferred into a fresh tube and the leaf material was re-extracted with $0.5 \mathrm{~mL}$ ethylacetate. The organic phases were pooled and evaporated to dryness. The dry residue was reconstituted in $0.4 \mathrm{~mL}$ of $70 / 30(\mathrm{v} / \mathrm{v})$ methanol/water for analysis with an LC-ESI-MS/MS instrument (Varian 1200 Triple-Quadrupole-LC-MS system; Varian, Palo Alto, CA). Ten $\mu \mathrm{L}$ of the sample were injected in a ProntoSIL ${ }^{\circledR}$ column (C18-ace-EPS, $50 \times 2 \mathrm{~mm}, 5 \mu \mathrm{m}$, $120 \AA$, Bischoff, Leonberg, Germany) connected to a precolumn (C18, 4 x 2 mm, Phenomenex, Torrance, CA). As mobile phases $0.05 \%$ formic acid in water (solvent $\mathrm{A}$ ) and methanol (solvent B) were used in a gradient mode with the following conditions: time/concentration $(\mathrm{min} / \%)$ for $\mathrm{B}$ : $0.0 / 15 ; 2.5 / 15 ; 4.5 / 98 ; 10.5 / 98 ; 12.0 / 15 ; 15.0 / 15$; time/flow (min/mL): 0.0/0.4; 1.5/0.2; 1.5/0.2; 10.5/0.4; 15.0/0.4. Compounds were detected in the ESI negative mode and multiple reaction monitoring (MRM) according to published parameters [49].

\section{Ethylene (ET) measurements}

ET levels were analyzed by photoacoustic spectrometry (INVIVO; https://www.invivo-gmbh.de) as previously described [50]. Corollas were excised from antherectomized flowers at different times (Additional file 3) and immediately placed in $100 \mathrm{~mL}$ sealed-glass vessels (four corollas per vessel) for $4.5 \mathrm{~h}$ before ET quantification. Three biological replicates per time and per genotype were performed. Antherectomization was performed in the morning before pollen release to avoid the production of ET induced by fertilization.

\section{Quantification of benzyl acetone (BA) emission from flowers}

Flower volatiles were collected by enclosing individual night flowers in plastic cups connected to Super-Q filter traps (ARS, Philadelphia, PA) under an air flow of $30 \mathrm{~mL} \mathrm{~min}^{-1}$. Volatiles were collected from $3 \mathrm{pm}$ to $9 \mathrm{am}$. Filter traps were spiked with $400 \mathrm{ng}$ of tetraline as an internal standard (IS) and eluted with $250 \mu \mathrm{L}$ of dichloromethane. Volatile analysis was performed by GC-MS with a CP-4000 GC instrument (Varian, Palo Alto, CA) on a DB-5 column 
(Agilent, Waldbronn, Germany). BA was identified by comparing retention times and mass spectra with a commercial BA standard (Sigma, Taufkirchen, Germany). Peak areas were integrated and normalized to the area of the IS.

BA was also analyzed by the $\mathrm{zNOSE}^{\mathrm{TM}}$ as previously described [51]. In this case, individual flowers were placed in scintillation vials and the vials were left capped. Every $2 \mathrm{~h}$ (starting at $6 \mathrm{pm}$; Figure $4 \mathrm{~d}$ ) the needle of the $\mathrm{zNOSE}^{\mathrm{TM}}$ was introduced into the vials to determine BA levels in the headspace. The vials were immediately capped again. The intensity of the peak area corresponding to BA was used to quantify BA levels per flower.

\section{Analysis of gene expression by qPCR}

Total RNA was extracted from different tissues by the TRIzol $^{\circledR}$ reagent (Invitrogen) and DNase-I treated (Fermentas, St. Leon-Rot, Germany) according to commercial instructions. Five $\mu \mathrm{g}$ of total RNA were reverse transcribed using oligo(dT)18 and SuperScript reverse transcriptase II (Invitrogen) according to commercial instructions. Quantitative real-time PCR (qPCR) was performed with the Mx3005P Multiplex qPCR system (Stratagene, La Jolla, CA) and the qPCR Core kit for SYBR $^{\circledR}$ Green I (Eurogentec, Liege, Belgium) using gene specific primers (Additional file 6). Quantification of HD20 mRNA levels was performed by normalization with the Elongation Factor 1A (EF1A) mRNA according to the $\Delta \mathrm{Ct}$ method [52]. All the reactions were performed with at least three biological replicates.

\section{Microarray analysis}

Corollas from ir-hd20 and WT plants were collected at $12 \mathrm{pm}$ (a total of three independent samples (biological replicates) were used per genotype and each sample was composed of three pooled corollas). Total RNA was extracted from corollas based on a previously described method [53] and its quality was checked by spectrophotometry (NanoDrop, Wilmington, DE). Genomic DNA was removed by DNAse treatment following commercial instructions (Turbo DNase; Ambion, Europe), RNA was cleaned up with RNeasy MinElute columns (Qiagen, Hilden, Germany) and the RNA quality was checked with the RNA 6000 n kit (Agilent, Santa Clara, CA) using an Agilent 2100 Bioanalyzer. Total RNA was used to generate labeled cRNA with the Quick Amp labeling kit (Agilent) and the yield was determined spectrophotometrically (NanoDrop). Labeled cRNA was hybridized using the gene expression hybridization kit (Agilent) following commercial instructions onto $44 \mathrm{~K}$ custom-designed 60-mer $N$. attenuata Agilent microarray containing 43,533 sequences (see accession numbers)[28,29]. Microarrays were hybridized overnight at $65^{\circ} \mathrm{C}$ and slides were washed with the Gene Expression Wash Buffer kit (Agilent) as outlined in the One-Color Microarray-Based Gene Expression Analysis manual (Agilent). Three biological replicates were used per treatment with a total of six arrays. Arrays were scanned with an Agilent G2565BA scanner and image data was acquired with the Agilent Scan Control software (version A.7.0.1 for the B scanner). Data was extracted using the Agilent Feature Extraction software (version 9.5) and analyzed with the SAM (Significance Analysis of Microarrays) software [54]. The q-values for each gene corresponded to a computed false discovery rate (FDR) of $5.2 \%$. Significant changes in gene expression were considered when the fold change (FC; ir- $h d 20$ versus WT) were equal or greater than 1.9 or equal or smaller than 0.6 (with q-values lower than 0.048; according to the FDR value calculated by SAM). Gene Ontology (GO) and enzyme code (EC) classification and sequence analysis was performed with the Blast2GO software [55].

\section{Statistical analysis}

Statistics were calculated using the SPSS software version 17.0. The data was subjected either to one-way analysis of variance (ANOVA; and means were compared by the lowest standard deviation (LSD) test) or to Student's $t$-test as indicated in the text. The number of replicates $(n)$ used in each experiment are detailed in the figure's captions.

\section{Accession numbers}

Sequence data from this article can be found under the following accession numbers: HD20 [GenBank:HM107874], CHAL1 [GenBank:EU503226], FDR [GenBank:JQ028693], PP2C [GenBank:JQ028694], SAMT/BAMT [GenBank: JQ028692], Agilent Chip platform [NCBI GEO:GPL13527], and microarray data [NCBI GEO: GSE33682].

\section{Additional files}

Additional file 1: Southern blot analysis of ir-hd20 plants.

Additional file 2: Morphological and developmental characterization of ir-hd20 plants.

Additional file 3: Quantification of phytohormone levels in corollas of irhd20 and WT plants during corolla opening.

Additional file 4: Analysis of phytohormones in whole flowers of ir-hd20 and WT plants at different developmental stages.

Additional file 5: List of genes changing expression in corollas of irhd20 plants compared to WT plants.

Additional file 6: List of primers for $\mathrm{QPCR}$ analysis.

\section{Acknowledgements}

The DAAD (fellowship to DR), the ERASMUS Program (fellowship to BR) and the Max Planck Society are acknowledged for funding.

Author details

${ }^{1}$ Department of Molecular Ecology, Max Planck Institute for Chemical Ecology, 07745 Jena, Germany. ${ }^{2}$ Instituto de Agrobiotecnología del Litoral, 
Universidad Nacional del Litoral, CONICET, CC 242 Ciudad Universitaria, 3000 Santa Fe, Argentina.

\section{Authors' contributions}

DAR, BR and GB carried out the experiments and analyzed the data. ITB and RLC participated in the design of the study and edited the manuscript. GB conceived of the study and participated in its design and coordination. DAR and GB drafted the manuscript. All authors read and approved the final manuscript.

Received: 3 March 2012 Accepted: 1 May 2012

Published: 1 May 2012

\section{References}

1. Dudareva N, Pichersky E: Biochemical and molecular genetic aspects of floral scents. Plant Physiol 2000, 122(3):627-633.

2. Kessler D, Gase K, Baldwin IT: Field experiments with transformed plants reveal the sense of floral scents. Science 2008, 321(5893):1200-1202.

3. Kessler D, Baldwin IT: Making sense of nectar scents: the effects of nectar secondary metabolites on floral visitors of Nicotiana attenuata. Plant J 2007, 49(5):840-854

4. Raguso RA: Flowers as sensory billboards: progress towards an integrated understanding of floral advertisement. Curr Opin Plant Biol 2004, 7(4):434-440.

5. Spitzer-Rimon B, Marhevka E, Barkai O, Marton I, Edelbaum O, Masci T, Prathapani NK, Shklarman E, Ovadis M, Vainstein A: EOBII, a gene encoding a flower-specific regulator of phenylpropanoid volatiles' biosynthesis in petunia. Plant Cell 2010, 22(6):1961-1976.

6. Verdonk JC, Haring MA, van Tunen AJ, Schuurink RC: ODORANT1 regulates fragrance biosynthesis in petunia flowers. Plant Cell 2005,

17(5):1612-1624.

7. Pichersky E, Dudareva N: Scent engineering: toward the goal of controlling how flowers smell. Trends Biotechnol 2007, 25(3):105-110.

8. Dudareva N, Martin D, Kish CM, Kolosova N, Gorenstein N, Faldt J, Miller B, Bohlmann J: (E)-beta-ocimene and myrcene synthase genes of floral scent biosynthesis in snapdragon: function and expression of three terpene synthase genes of a new terpene synthase subfamily. Plant Cell 2003, 15(5):1227-1241.

9. Martin C, Gerats T: Control of Pigment Biosynthesis Genes during Petal Development. Plant Cell 1993, 5(10):1253-1264.

10. Euler $M$, Baldwin IT: The chemistry of defense and apparency in the corollas of Nicotiana attenuata. Oecologia 1996, 107(1):102-112.

11. Sime KR, Baldwin IT: Opportunistic out-crossing in Nicotiana attenuata (Solanaceae), a predominantly self-fertilizing native tobacco. BMC ECol 2003, 3:6.

12. Baldwin IT, Preston C, Euler M, Gorham D: Patterns and consequences of benzyl acetone floral emissions from Nicotiana attenuata plants. 1997, 23(10):2327-2343. doi:101007/s10886-007-9321-2.

13. Ariel FD, Manavella PA, Dezar CA, Chan RL: The true story of the HD-Zip family. Trends Plant Sci 2007, 12(9):419-426.

14. Sessa G, Morelli G, Ruberti I: DNA-binding specificity of the homeodomainleucine zipper domain. J Mol Biol 1997, 274(3):303-309.

15. Palena CM, Gonzalez DH, Chan RL: A monomer-dimer equilibrium modulates the interaction of the sunflower homeodomain leucine-zipper protein Hahb-4 with DNA. Biochem J 1999, 341(Pt 1):81-87.

16. Carabelli M, Sessa G, Baima S, Morelli G, Ruberti I: The Arabidopsis Athb-2 and -4 genes are strongly induced by far-red-rich light. Plant J 1993, 4(3):469-479.

17. Soderman E, Mattsson J, Engstrom P: The Arabidopsis homeobox gene ATHB-7 is induced by water deficit and by abscisic acid. Plant J 1996 , 10(2):375-381.

18. Soderman E, Hjellstrom M, Fahleson J, Engstrom P: The HD-Zip gene ATHB6 in Arabidopsis is expressed in developing leaves, roots and carpels and up-regulated by water deficit conditions. Plant Mol Biol 1999, 40(6):1073-1083.

19. Henriksson $E$, Olsson AS, Johannesson $H$, Johansson H, Hanson J, Engstrom P, Soderman E: Homeodomain leucine zipper class I genes in Arabidopsis.
Expression patterns and phylogenetic relationships. Plant Physiol 2005, 139(1):509-518.

20. Olsson AS, Engstrom P, Soderman E: The homeobox genes ATHB12 and ATHB7 encode potential regulators of growth in response to water deficit in Arabidopsis. Plant Mol Biol 2004, 55(5):663-677.

21. Lee YH, Chun JY: A new homeodomain-leucine zipper gene from Arabidopsis thaliana induced by water stress and abscisic acid treatment. Plant Mol Biol 1998, 37(2):377-384.

22. Johannesson $H$, Wang $Y$, Hanson J, Engstrom P: The Arabidopsis thaliana homeobox gene ATHB5 is a potential regulator of abscisic acid responsiveness in developing seedlings. Plant Mol Biol 2003, 51(5):719-729

23. Mayda E, Tornero P, Conejero V, Vera P: A tomato homeobox gene (HDzip) is involved in limiting the spread of programmed cell death. Plant J 1999, 20(5):591-600.

24. Lin Z, Hong Y, Yin M, Li C, Zhang K, Grierson D: A tomato HD-Zip homeobox protein, LeHB-1, plays an important role in floral organogenesis and ripening. Plant J 2008, 55(2):301-310

25. Deng X, Phillips J, Brautigam A, Engstrom P, Johannesson H, Ouwerkerk PB, Ruberti I, Salinas J, Vera P, lannacone R, et al: A homeodomain leucine zipper gene from Craterostigma plantagineum regulates abscisic acid responsive gene expression and physiological responses. Plant Mol Biol 2006, 61(3):469-489.

26. Harris JC, Hrmova M, Lopato S, Langridge P: Modulation of plant growth by HD-Zip class I and II transcription factors in response to environmental stimuli. New Phytol 2011, 190(4):823-837.

27. Re DA, Dezar CA, Chan RL, Baldwin IT, Bonaventure G: Nicotiana attenuata $\mathrm{NaHD2O}$ plays a role in leaf ABA accumulation during water stress, benzylacetone emission from flowers, and the timing of bolting and flower transitions. 2011, 62(1):155-166. 101093/jxb/erm035.

28. Kallenbach M, Gilardoni PA, Allmann S, Baldwin IT, Bonaventure G: C(12) derivatives of the hydroperoxide lyase pathway are produced by product recycling through lipoxygenase-2 in Nicotiana attenuata leaves. New Phytol 2011, 191(4):1054-1068.

29. Gilardoni PA, Hettenhausen C, Baldwin IT, Bonaventure G: Nicotiana attenuata LECTIN RECEPTOR KINASE1 suppresses the insect-mediated inhibition of induced defense responses during Manduca sexta herbivory. Plant Cell 2011, 23(9):3512-3532.

30. Devoto A, Nieto-Rostro M, Xie DX, Ellis C, Harmston R, Patrick E, Davis J, Sherratt L, Coleman M, Turner JG: COI1 links jasmonate signalling and fertility to the SCF ubiquitin-ligase complex in Arabidopsis. Plant J 2002, 32(4):457-466.

31. Paschold A, Halitschke R, Baldwin I: Co(i)-ordinating defenses: NaCOI1 mediates herbivore- induced resistance in Nicotiana attenuata and reveals the role of herbivore movement in avoiding defenses. Plant J 2007, 51(1):79-91.

32. Li L, Zhao YF, MCCaig BC, Wingerd BA, Wang JH, Whalon ME, Pichersky $E$, Howe GA: The tomato homolog of CORONATINE-INSENSITIVE1 is required for the maternal control of seed maturation, jasmonatesignaled defense responses, and glandular trichome development. Plant Cell 2004, 16(1):126-143.

33. Arce AL, Raineri J, Capella M, Cabello JV, Chan RL: Uncharacterized conserved motifs outside the HD-Zip domain in HD-Zip subfamily I transcription factors; a potential source of functional diversity. BMC Plant Biol 2011, 11:42.

34. Boudsocq M, Barbier-Brygoo H, Lauriere C: Identification of nine sucrose nonfermenting 1-related protein kinases 2 activated by hyperosmotic and saline stresses in Arabidopsis thaliana. 2004, 279(40):41758-41766. 101074/jbcM705780200.

35. Raghavendra AS, Gonugunta VK, Christmann A, Grill E: ABA perception and signalling. Trends Plant Sci 2010, 15(7):395-401.

36. Winkel-Shirley B: Flavonoid biosynthesis. A colorful model for genetics, biochemistry, cell biology, and biotechnology. Plant Physiol 2001, 126(2):485-493.

37. Colquhoun TA, Kim JY, Wedde AE, Levin LA, Schmitt KC, Schuurink RC, Clark DG: PhMYB4 fine-tunes the floral volatile signature of Petunia $x$ hybrida through PhC4H. 2011, 62(3):1133-1143. 101093/jxb/erm035. 
38. Cabello JV, Arce AL, Chan RL: The homologous HD-Zip I transcription factors HaHB1 and AtHB13 confer cold tolerance via the induction of pathogenesis-related and glucanase proteins. Plant J 2011, 69(1):141-153.

39. Park CJ, Peng Y, Chen X, Dardick C, Ruan D, Bart R, Canlas PE, Ronald PC: Rice $X B 15$, a protein phosphatase $2 C$, negatively regulates cell death and XA21-mediated innate immunity. PLoS Biology 2008, 6(9):e231.

40. Klingler JP, Batelli G, Zhu JK: ABA receptors: the START of a new paradigm in phytohormone signalling. 2010, 61(12):3199-3210. 101093/jxb/erm035.

41. Hirayama T, Shinozaki K: Perception and transduction of abscisic acid signals: keys to the function of the versatile plant hormone $A B A$. Trends Plant Sci 2007, 12(8):343-351.

42. Finkelstein RR, Somerville CR: Three Classes of Abscisic Acid (ABA)Insensitive Mutations of Arabidopsis Define Genes that Control Overlapping Subsets of ABA Responses. Plant Physiol 1990, 94(3):1172-1179.

43. Nelson DC, Lasswell J, Rogg LE, Cohen MA, Bartel B: FKF1, a clockcontrolled gene that regulates the transition to flowering in Arabidopsis. Cell 2000, 101(3):331-340

44. Xie DX, Feys BF, James S, Nieto-Rostro M, Turner JG: COI1: An Arabidopsis gene required for jasmonate-regulated defense and fertility. Science 1998, 280(5366):1091-1094.

45. von Dahl CC, Winz RA, Halitschke R, Kuhnemann F, Gase K, Baldwin IT: Tuning the herbivore-induced ethylene burst: the role of transcript accumulation and ethylene perception in Nicotiana attenuata. Plant J 2007, 51(2):293-307.

46. von Dahl CC, Baldwin IT: Deciphering the role of ethylene in plantherbivore interactions. Journal of Plant Growth Regulation 2007, 26(2):201-209.

47. Krügel T, Lim M, Gase K, Halitschke R, Baldwin IT: Agrobacterium-mediated transformation of Nicotiana attenuata, a model ecological expression system. Chemoecology 2002, 12(4):177-183.

48. Gase K, Weinhold A, Bozorov T, Schuck S, Baldwin IT: Efficient screening of transgenic plant lines for ecological research. Molecular Ecology Resources 2011, 11(5):890-902.

49. Bonaventure G, Schuck S, Baldwin IT: Revealing complexity and specificity in the activation of lipase-mediated oxylipin biosynthesis: a specific role of the Nicotiana attenuata GLA1 lipase in the activation of jasmonic acid biosynthesis in leaves and roots. Plant Cell Environ 2011, 34(9):1507-1520.

50. Körner E, von Dahl C, Bonaventure G, Baldwin IT: Pectin methylesterase NaPME1 contributes to the emission of methanol during insect herbivory and to the elicitation of defence responses in Nicotiana. 2009, 60:2631-2640. 101093/jxb/erm035.

51. Kessler D, Diezel C, Baldwin IT: Changing pollinators as a means of escaping herbivores. Curr Biol 2010, 20(3):237-242.

52. Gilardoni $P$, Schuck $S$, Jüngling R, Rotter B, Baldwin I, Bonaventure G: SuperSAGE analysis of the Nicotiana attenuata transcriptome after fatty acid-amino acid elicitation (FAC): identification of early mediators of insect responses. BMC Plant Biol 2010, 10:66

53. Kistner C, Matamoros M: RNA isolation using phase extraction and $\mathrm{LiCl}$ precipitation. In Lotus japonicus Handbook. Edited by Márquez A. Netherlands: Springer; 2005:123-124.

54. Tusher VG, Tibshirani R, Chu G: Significance analysis of microarrays applied to the ionizing radiation response. Proc Natl Acad Sci U S A 2001 98(9):5116-5121.

55. Conesa A, Gotz S, Garcia-Gomez JM, Terol J, Talon M, Robles M: Blast2GO: a universal tool for annotation, visualization and analysis in functional genomics research. Bioinformatics 2005, 21(18):3674-3676.

doi:10.1186/1471-2229-12-60

Cite this article as: Ré et al:: RNAi-mediated silencing of the HD-Zip gene HD20 in Nicotiana attenuata affects benzyl acetone emission from corollas via ABA levels and the expression of metabolic genes. $B M C$ Plant Biology 2012 12:60.

\section{Submit your next manuscript to BioMed Central and take full advantage of:}

- Convenient online submission

- Thorough peer review

- No space constraints or color figure charges

- Immediate publication on acceptance

- Inclusion in PubMed, CAS, Scopus and Google Scholar

- Research which is freely available for redistribution

Submit your manuscript at www.biomedcentral.com/submit 